Commun. Korean Math. Soc. 29 (2014), No. 2, pp. 311-317

http://dx.doi.org/10.4134/CKMS.2014.29.2.311

\title{
ASCREEN LIGHTLIKE HYPERSURFACES OF A SEMI-RIEMANNIAN SPACE FORM WITH A SEMI-SYMMETRIC NON-METRIC CONNECTION
}

\author{
Dae Ho Jin
}

\begin{abstract}
We study lightlike hypersurfaces of a semi-Riemannian space form $\widetilde{M}(c)$ admitting a semi-symmetric non-metric connection. First, we construct a type of lightlike hypersurfaces according to the form of the structure vector field of $\widetilde{M}(c)$, which is called a ascreen lightlike hypersurface. Next, we prove a characterization theorem for such an ascreen lightlike hypersurface endow with a totally geodesic screen distribution.
\end{abstract}

\section{Introduction}

The theory of lightlike submanifolds is an important topic of research in differential geometry due to its application in mathematical physics, especially in the electromagnetic field theory. The study of such notion was initiated by Duggal and Bejancu [3] and later studied by many authors (see up-to date results in two books $[4,5]$ ). The notion of a semi-symmetric non-metric connection on a Riemannian manifold was introduced by Ageshe and Chafle [1]. Recently several authors ([9]-[13]) studied lightlike hypersurfaces in a semi-Riemannian manifold admitting a semi-symmetric non-metric connection. Most of authors that wrote on either lightlike hypersurfaces $M$ of semi-Riemannian manifolds $\widetilde{M}$ admitting semi-symmetric non-metric connections or lightlike hypersurfaces $M$ of indefinite almost contact manifolds $\widetilde{M}$ fail to treat with the case the structure vector field $\zeta$ of $\widetilde{M}$ is not tangent to $M$, but studied only to the case $\zeta$ is tangent to $M$ (such $M$ is called tangential lightlike submanifold ([9]-[13]) of $\widetilde{M})$. There are few papers on non-tangential lightlike submanifolds of indefinite almost contact manifolds studied by Jin ([6]-[8]).

In this paper, we study non-tangential lightlike hypersurfaces of a semiRiemannian space form admitting a semi-symmetric non-metric connection. There are several different types of non-tangential lightlike hypersurfaces according to the form of the structure vector field of the ambient manifold. We

Received March 28, 2013.

2010 Mathematics Subject Classification. Primary 53C25, 53C40, 53C50.

Key words and phrases. totally geodesic, ascreen lightlike hypersurface, semi-symmetric non-metric connection. 
study a type of them here, named by ascreen lightlike hypersurfaces. Our main result is a classification theorem for such an ascreen lightlike hypersurface endow with a totally geodesic screen distribution.

\section{Semi-symmetric non-metric connection}

Let $(\widetilde{M}, \widetilde{g})$ be a semi-Riemannian manifold. A connection $\widetilde{\nabla}$ on $\widetilde{M}$ is called a semi-symmetric non-metric connection $[1]$ if $\widetilde{\nabla}$ and its torsion tensor $\widetilde{T}$ satisfy

$$
\begin{gathered}
\left(\widetilde{\nabla}_{X} \widetilde{g}\right)(Y, Z)=-\pi(Y) \widetilde{g}(X, Z)-\pi(Z) \widetilde{g}(X, Y), \\
\widetilde{T}(X, Y)=\pi(Y) X-\pi(X) Y,
\end{gathered}
$$

where $\pi$ is a 1 -form associated with a non-zero vector field $\zeta$ by

$$
\pi(X)=\widetilde{g}(X, \zeta),
$$

we say that $\zeta$ is the structure vector field of $\widetilde{M}$, for any vector fields $X, Y$ and $Z$ on $\widetilde{M}$. In the entire discussion of this article we shall assume that the structure vector field $\zeta$ to be unit spacelike unless otherwise specified.

Let $(M, g)$ be a lightlike hypersurface of $\widetilde{M}$. Then the normal bundle $T M^{\perp}$ of $M$ is a vector subbundle of the tangent bundle $T M$ of rank 1 , and coincides with the radical distribution $\operatorname{Rad}(T M)=T M \cap T M^{\perp}$ of $M$. Therefore there exist a complementary non-degenerate vector bundle $S(T M)$ of $\operatorname{Rad}(T M)$ in $T M$, which is called a screen distribution on $M$, such that

$$
T M=\operatorname{Rad}(T M) \oplus_{\text {orth }} S(T M),
$$

where $\oplus_{\text {orth }}$ denotes the orthogonal direct sum. We denote such a lightlike hypersurface by $M=(M, g, S(T M))$. Denote by $F(M)$ the algebra of smooth functions on $M$ and by $\Gamma(E)$ the $F(M)$ module of smooth sections of a vector bundle $E$ over $M$. It is well-known [3] that, for any null section $\xi$ of $\operatorname{Rad}(T M)$ on a coordinate neighborhood $\mathcal{U} \subset M$, there exists a unique null section $N$ of a unique vector bundle $\operatorname{tr}(T M)$ in $S(T M)^{\perp}$ satisfying

$$
\widetilde{g}(\xi, N)=1, \quad \widetilde{g}(N, N)=\widetilde{g}(N, X)=0, \quad \forall X \in \Gamma(S(T M) \mid \mathcal{u}) .
$$

We call $\operatorname{tr}(T M)$ and $N$ the transversal vector bundle and the null transversal vector field of $M$ with respect to $S(T M)$ respectively. Then $T \widetilde{M}$ is given by

$$
T \widetilde{M}=T M \oplus \operatorname{tr}(T M)=\{\operatorname{Rad}(T M) \oplus \operatorname{tr}(T M)\} \oplus_{\text {orth }} S(T M) .
$$

In the sequel, we take $X, Y, Z, W \in \Gamma(T M)$ unless otherwise specified. Let $P$ be the projection morphism of $T M$ on $S(T M)$. Then the local Gauss and Weingartan formulas for $M$ and $S(T M)$ are given, respectively, by

$$
\begin{aligned}
& \widetilde{\nabla}_{X} Y=\nabla_{X} Y+B(X, Y) N, \\
& \widetilde{\nabla}_{X} N=-A_{N} X+\tau(X) N ; \\
& \nabla_{X} P Y=\nabla_{X}^{*} P Y+C(X, P Y) \xi, \\
& \nabla_{X} \xi=-A_{\xi}^{*} X-\sigma(X) \xi,
\end{aligned}
$$


where $\nabla$ and $\nabla^{*}$ are the induced linear connections on $T M$ and $S(T M)$ respectively, $B$ and $C$ are the local second fundamental forms on $T M$ and $S(T M)$, respectively, $A_{N}$ and $A_{\xi}^{*}$ are the shape operators on $T M$ and $S(T M)$, respectively and $\tau$ is a 1-form on $T M$. From (2.1), (2.2) and (2.6), we have

$$
\begin{gathered}
\left(\nabla_{X} g\right)(Y, Z)=-\pi(Y) g(X, Z)-\pi(Z) g(X, Y) \\
+B(X, Y) \eta(Z)+B(X, Z) \eta(Y), \\
T(X, Y)=\pi(Y) X-\pi(X) Y,
\end{gathered}
$$

and $B$ is symmetric on $T M$, where $T$ is the torsion tensor with respect to the induced connection $\nabla$ and $\eta$ is a 1 -form on $T M$ such that

$$
\eta(X)=\widetilde{g}(X, N) .
$$

From the fact $B(X, Y)=\widetilde{g}\left(\widetilde{\nabla}_{X} Y, \xi\right)$, we know that $B$ is independent of the choice of a screen distribution. Taking $Y=\xi$ to this and using (2.1), we get

$$
B(X, \xi)=0 \text {. }
$$

Let $a$ and $b$ be the smooth functions defined by $a=\pi(N)$ and $b=\pi(\xi)$. Then the above second fundamental forms are related to their shape operators by

$$
\begin{aligned}
& g\left(A_{\xi}^{*} X, Y\right)=B(X, Y)-b g(X, Y), \quad \widetilde{g}\left(A_{\xi}^{*} X, N\right)=0, \\
& g\left(A_{N} X, P Y\right)=C(X, P Y)-a g(X, P Y)-\eta(X) \pi(P Y), \\
& \widetilde{g}\left(A_{N} X, N\right)=-a \eta(X), \quad \sigma(X)=\tau(X)-b \eta(X) .
\end{aligned}
$$

By (2.13), we show that $A_{\xi}^{*}$ is $S(T M)$-valued self-adjoint operator and satisfies

$$
A_{\xi}^{*} \xi=0 .
$$

Denote by $\widetilde{R}, R$ and $R^{*}$ the curvature tensors of the semi-symmetric nonmetric connection $\widetilde{\nabla}$ on $\widetilde{M}$, the induced connection $\nabla$ on $M$ and the induced connection $\nabla^{*}$ on $S(T M)$, respectively. Using the Gauss -Weingarten equations for $M$ and $S(T M)$, we obtain the Gauss-Codazzi equations for $M$ and $S(T M)$ :

$$
\begin{aligned}
& \widetilde{R}(X, Y) Z=R(X, Y) Z+B(X, Z) A_{N} Y-B(Y, Z) A_{N} X \\
& +\left[\left(\nabla_{X} B\right)(Y, Z)-\left(\nabla_{Y} B\right)(X, Z)+B(Y, Z)\{\tau(X)-\pi(X)\}\right. \\
& -B(X, Z)\{\tau(Y)-\pi(Y)\}] N, \\
& \widetilde{R}(X, Y) N=-\nabla_{X}\left(A_{N} Y\right)+\nabla_{Y}\left(A_{N} X\right)+A_{N}[X, Y]+\tau(X) A_{N} Y \\
& -\tau(Y) A_{N} X+\left\{B\left(Y, A_{N} X\right)-B\left(X, A_{N} Y\right)+2 d \tau(X, Y)\right\} N, \\
& R(X, Y) P Z=R^{*}(X, Y) P Z+C(X, P Z) A_{\xi}^{*} Y-C(Y, P Z) A_{\xi}^{*} X \\
& +\left[\left(\nabla_{X} C\right)(Y, P Z)-\left(\nabla_{Y} C\right)(X, P Z)+C(X, P Z)\{\sigma(Y)+\pi(Y)\}\right. \\
& -C(Y, P Z)\{\sigma(Y)+\pi(Y)\}] \xi \\
& R(X, Y) \xi=-\nabla_{X}^{*}\left(A_{\xi}^{*} Y\right)+\nabla_{Y}^{*}\left(A_{\xi}^{*} X\right)+A_{\xi}^{*}[X, Y]-\sigma(X) A_{\xi}^{*} Y \\
& +\sigma(Y) A_{\xi}^{*} X+\left\{C\left(Y, A_{\xi}^{*} X\right)-C\left(X, A_{\xi}^{*} Y\right)-2 d \sigma(X, Y)\right\} \xi .
\end{aligned}
$$

In case $R=0\left(R^{*}=0\right), M$ (the leaf $M^{*}$ of $\left.S(T M)\right)$ is called a flat manifold. 
A complete simply connected semi-Riemannian manifold $\widetilde{M}$ of constant curvature $c$ is called a semi-Riemannian space form and denote it by $\widetilde{M}(c)$. For any $X, Y, Z \in \Gamma(T \widetilde{M})$, the curvature tensor $\widetilde{R}$ of $\widetilde{M}(c)$ is given by

$$
\widetilde{R}(X, Y) Z=c\{\widetilde{g}(Y, Z) X-\widetilde{g}(X, Z) Y\} .
$$

In general, $S(T M)$ is not necessarily integrable. The following result gives equivalent conditions for the integrability of $S(T M)$ [9]:

Theorem 2.1. Let $M$ be a lightlike hypersurface of a semi-Riemannian manifold $\widetilde{M}$ admitting a semi-symmetric metric connection. Then the following assertions are equivalent:

(1) The screen distribution $S(T M)$ is an integrable distribution.

(2) $C$ is symmetric, i.e., $C(X, Y)=C(Y, X)$ for all $X, Y \in \Gamma(S(T M))$.

(3) The shape operator $A_{N}$ is self-adjoint with respect to $g$, i.e.,

$$
g\left(A_{N} X, Y\right)=g\left(X, A_{N} Y\right), \quad \forall X, Y \in \Gamma(S(T M)) .
$$

\section{Ascreen lightlike hypersurfaces}

Definition. A lightlike hypersurface $M$ of a semi-Riemannian manifold $\widetilde{M}$ admitting a semi-symmetric non-metric connection is called an ascreen lightlike hypersurface $[7,8]$ if $\zeta$ belongs to $S(T M)^{\perp}=\operatorname{Rad}(T M) \oplus \operatorname{tr}(T M)$.

If $M$ is an ascreen lightlike hypersurface of $\widetilde{M}$, then $\zeta$ is expressed as

$$
\zeta=a \xi+b N .
$$

As $\widetilde{g}(\zeta, \zeta)=1$, we have $2 a b=1$. This implies that $a \neq 0$ and $b \neq 0$. Taking the scaler product with $X$ to $(3.1)$, we have $\pi(X)=b \eta(X)$ for all $X \in \Gamma(T M)$. Comparing this with the third equation of (2.14), we obtain

$$
\tau(X)=\pi(X)+\sigma(X), \quad \forall X \in \Gamma(T M) .
$$

Theorem 3.1. Let $M$ be an ascreen lightlike hypersurface of a semi-Riemannian manifold $\widetilde{M}$ admitting a semi-symmetric non-metric connection. Then the screen distribution $S(T M)$ is an integrable distribution.

Proof. Taking the scalar product with $\xi$ to (2.17) and $N$ to (2.16) such that $Z=\xi$ by turns and using (2.12) and (2.19), we obtain

$$
\begin{aligned}
\widetilde{g}(\widetilde{R}(X, Y) \xi, N) & =B\left(X, A_{N} Y\right)-B\left(Y, A_{N} X\right)-2 d \tau(X, Y) \\
& =C\left(Y, A_{\xi}^{*} X\right)-C\left(X, A_{\xi}^{*} Y\right)-2 d \sigma(X, Y) .
\end{aligned}
$$

Substituting (2.13) and (2.14) into the last equation and using (3.2) and the facts $\pi\left(A_{\xi}^{*} X\right)=0$ for any $X \in \Gamma(T M)$ and $A_{\xi}^{*}$ is self-adjoint, we have

$$
2 d \pi(X, Y)=b\left\{g\left(X, A_{N} Y\right)-g\left(A_{N} X, Y\right)\right\}, \quad \forall X, Y \in \Gamma(T M) .
$$

As $\pi=0$ on $S(T M)$, we show that $d \pi=0$ on $S(T M)$. Thus we obtain

$$
g\left(A_{N} X, Y\right)=g\left(X, A_{N} Y\right), \quad \forall X, Y \in \Gamma(S(T M)) .
$$

It follows from Theorem 2.1 that $S(T M)$ is an integrable distribution. 
Definition. (1) A lightlike hypersurface $M$ is called totally umbilical [3] if there exist a smooth function $\beta$ on a coordinate neighborhood $\mathcal{U}$ in $M$ such that

$$
B(X, Y)=\beta g(X, Y), \forall X, Y \in \Gamma(T M) .
$$

In case $\beta=0$ on $\mathcal{U}$, we say that $M$ is totally geodesic.

(2) A screen distribution $S(T M)$ is called totally geodesic [3] in $M$ if $C=0$ on a coordinate neighborhood $\mathcal{U}$ in $M$.

Due to (2.14), we show that $S(T M)$ is totally geodesic in $M$ if and only if the shape operators $A_{N}$ of $S(T M)$ satisfies

$$
A_{N} X=-a X, \quad \forall X \in \Gamma(T M) .
$$

Theorem 3.2. Let $M$ be an ascreen lightlike hypersurface of a semi-Riemannian space form $\widetilde{M}(c)$ admitting a semi-symmetric non-metric connection. If $S(T M)$ is totally geodesic in $M$, then $c=0$ and $M$ is flat, totally geodesic and locally a product manifold $M=\mathcal{C} \times M^{*}$, where $\mathcal{C}$ is a null curve tangent to $\operatorname{Rad}(T M)$ and $M^{*}$ is a flat leaf of the integrable distribution $S(T M)$.

Proof. As $S(T M)$ is totally geodesic in $M$, from (2.8), (2.9) and (2.15), we show that $\operatorname{Rad}(T M)$ and $S(T M)$ are auto-parallel distributions of $M$. By de Rham's decomposition theorem [2], $M$ is locally a product manifold $\mathcal{C} \times M^{*}$ where $\mathcal{C}$ is a null curve tangent to $\operatorname{Rad}(T M)$ and $M^{*}$ is a leaf of $S(T M)$.

Taking the scalar product with $N$ to $(2.18)$, we obtain

$$
\widetilde{g}(R(X, Y) P Z, N)=0, \quad \forall X, Y, Z \in \Gamma(T M),
$$

due to $C=0$. Taking the scalar product with $N$ to $(2.16)$ such that $Z=P Z$ and using $(2.14)_{2},(2.20)$ and the last equation, we get

$$
c\{g(Y, P Z) \eta(X)-g(X, P Z) \eta(Y)\}=a\{B(Y, P Z) \eta(X)-B(X, P Z) \eta(Y)\} .
$$

Replacing $X$ by $\xi$ to this and using (2.12), we have

$$
c g(X, P Y)=a B(X, P Y), \quad \forall X, Y \in \Gamma(T M) .
$$

Using (2.12) and the fact $a \neq 0$, we obtain

$$
B(X, Y)=\beta g(X, Y), \quad \forall X, Y \in \Gamma(T M),
$$

where $\beta=c / a$. It follows that $M$ is totally umbilical.

Taking the scalar product with $\xi$ to $(2.16)$ and using (2.20), we have

$$
\begin{aligned}
& \left(\nabla_{X} B\right)(Y, Z)-\left(\nabla_{Y} B\right)(X, Z) \\
= & \{\pi(X)-\tau(X)\} B(Y, Z)-\{\pi(Y)-\tau(Y)\} B(X, Z) .
\end{aligned}
$$

Substituting $(3.4) \sim(3.6)$ into (2.16) and using (2.20) and $a \beta=c$, we have

$$
R(X, Y) Z=0, \quad \forall X, Y, Z \in \Gamma(T M) .
$$

Thus $M$ is a flat manifold. From this, (2.18) and the fact $C=0$, we also have

$$
R^{*}(X, Y) Z=0, \quad \forall X, Y, Z \in \Gamma(S(T M)) .
$$


Thus $M^{*}$ is also a flat manifold.

Taking the scalar product with $P Z$ to (2.17), we have

$$
\begin{aligned}
\widetilde{g}(\widetilde{R}(X, Y) N, P Z)= & g\left(-\nabla_{X}\left(A_{N} Y\right)+\nabla_{Y}\left(A_{N} X\right)+A_{N}[X, Y], P Z\right) \\
& +\tau(X) g\left(A_{N} Y, P Z\right)-\tau(Y) g\left(A_{N} X, P Z\right)
\end{aligned}
$$

for any $X, Y, Z \in \Gamma(T M)$. Using this, (2.20) and (3.4), we have

$$
\begin{aligned}
& c\{g(Y, P Z) \eta(X)-g(X, P Z) \eta(Y)\} \\
= & g\left(\nabla_{X}\left(A_{N} Y\right)-\nabla_{Y}\left(A_{N} X\right)-A_{N}[X, Y], P Z\right) \\
& +a\{\tau(X) g(Y, P Z)-\tau(Y) g(X, P Z)\}
\end{aligned}
$$

for any $X, Y, Z \in \Gamma(T M)$. Applying $\nabla_{X}$ to $A_{N} Y=-a Y$, we have

$$
\nabla_{X}\left(A_{N} Y\right)=-X[a] Y-a \nabla_{X} Y
$$

Substituting this into (3.7), we have

$$
\begin{aligned}
& \{X[a]-a \pi(X)-a \tau(X)+c \eta(X)\} g(Y, Z) \\
= & \{Y[a]-a \pi(Y)-a \tau(Y)+c \eta(Y)\} g(X, Z) .
\end{aligned}
$$

Applying $\nabla_{X}$ to $B(Y, Z)=\beta g(Y, Z)$ and using (2.10) and (3.5), we have

$$
\begin{gathered}
\left(\nabla_{X} B\right)(Y, Z)=\quad X[\beta] g(Y, Z)-\left\{\beta \pi(Y)-\beta^{2} \eta(Y)\right\} g(X, Z) \\
-\left\{\beta \pi(Z)-\beta^{2} \eta(Z)\right\} g(X, Y) .
\end{gathered}
$$

Substituting this equation into (3.6), we get

$$
\left\{X[\beta]+\beta \tau(X)-\beta^{2} \eta(X)\right\} g(Y, Z)=\left\{Y[\beta]+\beta \tau(Y)-\beta^{2} \eta(Y)\right\} g(X, Z) .
$$

Replacing $\beta$ by $c / a$ to this equation and using the fact $a \neq 0$, we have

$$
c\{X[a]-a \tau(X)+c \eta(X)\} g(Y, Z)=c\{Y[a]-a \tau(Y)+c \eta(Y)\} g(X, Z) .
$$

Now we assume that $c \neq 0$. Then, for any $X, Y, Z \in \Gamma(T M)$, we have

$$
\{X[a]-a \tau(X)+c \eta(X)\} g(Y, Z)=\{Y[a]-a \tau(Y)+c \eta(Y)\} g(X, Z) .
$$

Comparing (3.8) and (3.9) and using the fact $a \neq 0$, we have

$$
\pi(X) g(Y, Z)=\pi(Y) g(X, Z), \quad \forall X, Y, Z \in \Gamma(T M) .
$$

Taking $Y=Z=P W$ such that $g(P W, P W) \neq 0$ on $\mathcal{U}$ and by using the fact $\pi(P W)=0$, we obtain $\pi(X)=0$ for all $X \in \Gamma(T M)$. It is a contradiction to $\pi(\xi)=b \neq 0$. It follows that $c=0$. Therefore $\beta=0$. By (3.5), we show that $M$ is totally geodesic. Thus we have our assertion. 


\section{References}

[1] N. S. Agashe and M. R. Chafle, A semi-symmetric non-metric connection on a Riemannian manifold, Indian J. Pure Appl. Math. 23 (1992), no. 6, 399-409.

[2] G. de Rham, Sur la réductibilité d'un espace de Riemannian, Comment. Math. Helv. 26 (1952), 328-344.

[3] K. L. Duggal and A. Bejancu, Lightlike Submanifolds of Semi-Riemannian Manifolds and Applications, Kluwer Acad. Publishers, Dordrecht, 1996.

[4] K. L. Duggal and D. H. Jin, Null curves and Hypersurfaces of Semi-Riemannian Manifolds, World Scientific, 2007.

[5] K. L. Duggal and B. Sahin, Differential Geometry of Lightlike Submanifolds, Frontiers in Mathematics, Birkhäuser, 2010.

[6] D. H. Jin, Transversal half lightlike submanifolds of an indefinite Sasakian manifold, J. Korea Soc. Math. Educ. Ser. B Pure Appl. Math. 18 (2011), no. 1, 51-61.

[7] _ Special half lightlike submanifolds of an indefinite cosymplectic manifold, J. Funct. Spaces Appl. 2012 (2012), Art. ID 636242, 16 pp.

[8] _ Ascreen lightlike hypersurfaces of an indefinite Sasakian manifold, J. Korean Soc. Math. Educ. Ser. B Pure Appl. Math. 20 (2013), no. 1, 25-35.

[9] — Geometry of lightlike hypersurfaces of a semi-Riemannian space form with a semi-symmetric non-metric connection, submittied in Indian J. Pure Appl. Math.

[10] _ Einstein lightlike hypersurfaces of a Lorentz space form with a semi-symmetric non-metric connection, accepted in Bull. Korean Math. Soc.

[11] _ Two characterization theorems for lightlike geometry, submitted in Honam Math. J.

[12] _ A classification of screen quasi-conformal Einstein lightlike hypersurfaces of a semi-Riemannian space form with a semi-symmetric non-metric connection, submitted in J. Fun. Sp. Math.

[13] E. Yasar, A. C. Cöken, and A. Yücesan, Lightlike hypersurfaces in semi-Riemannian manifold with semi-symmetric non-metric connection, Math. Scand. 102 (2008), no. 2, $253-264$.

Department of Mathematics

DONGGUK UNIVERSITY

Gyeonguu 780-714, Korea

E-mail address: jindh@dongguk.ac.kr 